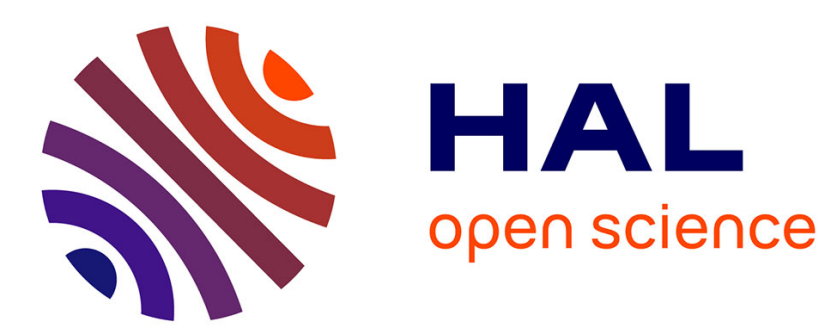

\title{
A Causal Graph Based Method to Evaluate Ecollaboration Scenarios
}

\author{
Raoudha Chebil, Wided Lejouad-Chaari, Stefano A. Cerri, Khaled Ghedira
}

\section{To cite this version:}

Raoudha Chebil, Wided Lejouad-Chaari, Stefano A. Cerri, Khaled Ghedira. A Causal Graph Based Method to Evaluate Ecollaboration Scenarios. WETICE: Workshops on Enabling Technologies - Infrastracture for Collaborative Enterprises, Jun 2013, Hammamet, Tunisia. pp.225-230, 10.1109/WETICE.2013.10 . lirmm-00822885

\section{HAL Id: lirmm-00822885 https://hal-lirmm.ccsd.cnrs.fr/lirmm-00822885}

Submitted on 15 Jun 2013

HAL is a multi-disciplinary open access archive for the deposit and dissemination of scientific research documents, whether they are published or not. The documents may come from teaching and research institutions in France or abroad, or from public or private research centers.
L'archive ouverte pluridisciplinaire HAL, est destinée au dépôt et à la diffusion de documents scientifiques de niveau recherche, publiés ou non, émanant des établissements d'enseignement et de recherche français ou étrangers, des laboratoires publics ou privés. 


\section{A Causal Graph Based Method to Evaluate E- collaboration Scenarios}

\author{
Raoudha CHEBIL, Wided LEJOUAD CHAARI \\ Laboratory of Strategies of Optimization and Intelligent \\ Computing (SOIE) \\ National School of Computer Studies (ENSI) - Manouba \\ University \\ Campus de la Manouba, 2010 Manouba, Tunisia \\ raoudha.chebil,wided.chaari@ensi.rnu.tn
}

\author{
Stefano. A CERRI
}

The Montpellier Laboratory of Informatics, Robotics, and Microelectronics (LIRMM)

\begin{abstract}
Electronic collaboration is a pervasive concept that influences practically all human domains, from work to learning to entertainment; making possible the collaboration between two or more distant persons. To encourage the exploitation of this new phenomenon, we have to demonstrate its benefits using validated evaluating methods that ensure the reliability of its effects. However, e-collaboration environments are not just classical human-computer interfaces: the criteria for their evaluation include teleological properties like the collaboration goal, the tasks and also particular constraints like the connectivity, the used soft platform, the quality of services and the behavior of the collaboration members. Our research work attempts to propose a reusable e-collaboration evaluation method. To attain this goal, we began by carrying out some abstractions about e-collaboration sessions giving a more rigorous vision about these environments. Once this step accomplished, the evaluation work based on the previously cited vision can be started.
\end{abstract}

Keywords - E-collaboration; Performance evaluation; Scenario based analysis; Modeling.

\section{INTRODUCTION}

Generally, collaboration involves attractive exchanges and many motivated efforts producing results more interesting than individual work. By definition, this concept requires its participants to be in the same geographical site at the same time. But such constraint can't be accepted in the current ages of technological emergence that has broken all geographical and temporal barriers. Frequently two or more persons in different ends of the world have common interests and need to collaborate. This requirement must not be dropped due to its consequent constraints; it has to be supported by existing technologies to help participants producing interesting results promoting progress in the concerned domain. Today, a wide range of collaborative platforms is available offering services more and more sophisticated and adapted to all the needs. Despite all this technological wealth in perpetual growth, its exploitation is still limited and slowed by a poor reliability level. Unfortunately, in many cases, we notice that in order to attend a meeting of few hours; some people prefer spending

\author{
University of Montpellier $2 \&$ the National Center for \\ Scientific Research \\ 161, Rue Ada - F-34095 Montpellier Cedex 5, France \\ stefano.cerri@lirmm.fr \\ Khaled GHEDIRA \\ Laboratory of Strategies of Optimization and Intelligent \\ Computing (SOIE) \\ High School of Management (ISG) - University of Tunis \\ 41, Rue de la liberté, Cité Bouchoucha 2000, \\ Le Bardo, Tunisia \\ khaled.ghedira@isg.rnu.tn
}

time and money in travelling instead of using e-collaboration tools. It is true that passing from a real to a virtual environment, involves the introduction of some technological and psychological factors that could have, sometimes, negative effects on the collaborative work progress. Even if this risk is low, in high concurrency environments it can't be neglected; this explains the preference of classical meetings to virtual ones. To change the described behavior face to ecollaboration tools, we estimate necessary to resolve the existing reliability problem by evaluating rigorously these environments performances and progressively improve them.

The existing works on e-collaboration performance evaluation were not sufficient to reassure users as some of them are related to particular application domains and others present new ideas without any validation. Many works on ecollaboration are based on abstractions and present very theoretical ideas making them far from reality and difficult to apply to real cases. These findings motivated us to propose a new evaluating approach supposed to be easier to apply and based on the analysis of real scenarios.

This paper will be organized as follows. In section 2 , we position the reader in the context by summarizing most of the existing work on e-collaboration. In section 3 , we present the different studied evaluation alternatives and we prove the choice of the proposed approach. In section 4, we describe the carried out scenario based analysis as well as the obtained results. In section 5, we present the proposed evaluation method based on the previously outlined abstractions and composed of two levels. Finally, in section 6, we explain more our method by applying it on a test scenario.

\section{STATE OF THE ART}

The beginnings of e-collaboration date from mid-1800s when the telegraph was invented. But this new tool wasn't sufficiently practical to diffuse largely the concept. The same occurred for the telephone in 1870 . With the advent of the first commercial computers known under the name of mainframes, e-collaboration emerged and was more favored 
by the e-mail development. Afterwards, the creation of Wide Area Networks (WAN) and the development of personal computers encouraged the design of new technologies and platforms for this recent concept. Consequently, many key words appeared like: CMC (Computer Mediated Communication) and $\mathrm{CSCW}$ (Computer Supported Collaborative Work) as new concepts and GDSS (Group Decision Support Systems) as a new mean enabling the increase of meeting efficiency. Many platforms were available like: Groupsystems, Teamfocus and Lotus Notes. The first research on e-collaboration were launched in the 70ties giving birth to the first papers on e-collaboration in the mid-1990s. The main topics consisted in finding technological solutions for e-collaboration problems and analyzing tool effects on group behavior in e-collaboration environments. These topics evolved over time and some of them were resolved while new ones appeared. In particular, performance evaluation was a research item treated by many works and it isn't resolved till now [10].

In the literature, many works propose performance evaluation methods for e-collaboration environments. They classify evaluations in four types according to their aim [2]: feasibility evaluation that is based on the cost, iterative evaluation that aims to improve collaborative platforms, comparative evaluation that compares systems and appropriateness evaluation that determines if a system is appropriate to a given organization's process.

Generally, in considered works, evaluation is carried out by:

- Identifying significant metrics and measuring them [1].

- Designing questionnaires reflecting the aspects to evaluate and exploiting them [2].

We note from the literature study the multiplicity of dispersed works generally without any validation [6][7]. This explains the lack of standards for performance evaluation and the frequency of subjective statements on e-collaboration performance. The absence of rigorous evaluation works will not favor the concept improvement and its reliability. This problem has motivated the presented work aiming to propose an e-collaboration evaluation method.

\section{PROPOSED APPROACH}

Performance Evaluation is a wide term that concerns different works focused in varied aspects depending on the considered system as well as the aimed performance criteria. Our contribution has started with a study of different possible alternatives of evaluation and the choice of the most adapted approach to the e-collaboration context. The studied evaluation ideas can be summarized in the following points:

- Focus on the communication quality aspect by using formulas of networks performance evaluation;

- Consider only three important aspects in evaluation: communication support, interface and collaborators behavior;

- Start by an analysis of real e-collaboration scenarios permitting to produce general results representing all e-collaboration scenarios. These results are used to find a reusable evaluation solution.
We estimate that the first idea is not applicable to the considered scenarios which present many additional characteristics compared to simple networks. In fact, in machine networks, the computing speed and precision as well as the response time to requests are significant. But it is not the same case when the network's nodes are constituted by humans who collaborate under particular constraints aiming to attain some objectives.

For the second idea, despite the importance of previously cited aspects, we estimate that e-collaboration evaluation can't be reduced to a combination of separate network, Human-Machine-Interface and behavior evaluations as the whole is greater than the sum of parts.

The third idea is to start with an analysis of several real ecollaboration scenarios followed by successive abstractions permitting to produce a general model representing all ecollaboration scenarios. The proposed evaluation method would be based on the obtained model. This alternative seems more realizable and adequate to e-collaboration environments that are various, dynamic and strongly dependent on their contexts including objectives, progress conditions and the unpredictable human behavior.

\section{SCENARIO BASED ANALYSIS}

The evaluation task consists mainly on comparing obtained results with expected ones taking into account the environment's characteristics and constraints. This strong context's dependence makes the evaluation task more difficult; so it is inappropriate to use the classical and well known performance aspects like computing time results and accuracy to describe e-collaboration quality. Face to this subjective and particular environment, our idea consists in starting with an analysis of some real e-collaboration scenarios in order to detect their common aspects and carry out some abstractions to be able to product reusable results. To be more concrete, we present in the following, three examples of the considered scenarios, belonging to different contexts.

\section{A. Scenario examples}

The first scenario is part of ELeGI (European Learning Grid Infrastructure) project involving students having to edit, design and produce a Spanish-language web-based newsletter, containing items of interest to the Spanishlanguage community. These collaboration members, have to decide what the scope of the content should actually be (e.g. sports, news, culture). Each group of four students is supervised by a tutor directing them in the different required tasks and evaluating their works at the end. Communications between participants are ensured by BuddySpace services [4].

The second scenario aims to improve the business modeling language entitled BPMN (Business Process Modeling Notation).

For each detected representation weakness or problem, the project participants, launch a discussion and collect proposed solutions, then an e-mail vote process is carried out to determine what solution will be retained [5].

The third scenario is supported by the Flashmeeting videoconferencing environment and consists of a virtual 
meeting of a research team chaired by its director in order to supervise the work progress of each member and to discuss faced problems. A defined time is accorded to each researcher to present his recent results, to explain encountered difficulties and to discuss with other members about the future work.

\section{B. General model}

Despite their diversity, all e-collaboration scenarios include the following common components summarized in Fig.1:

- An e-collaboration platform;

- Participants to e-collaboration named e-collaborators;

- Sub-goals;

- Global goals.

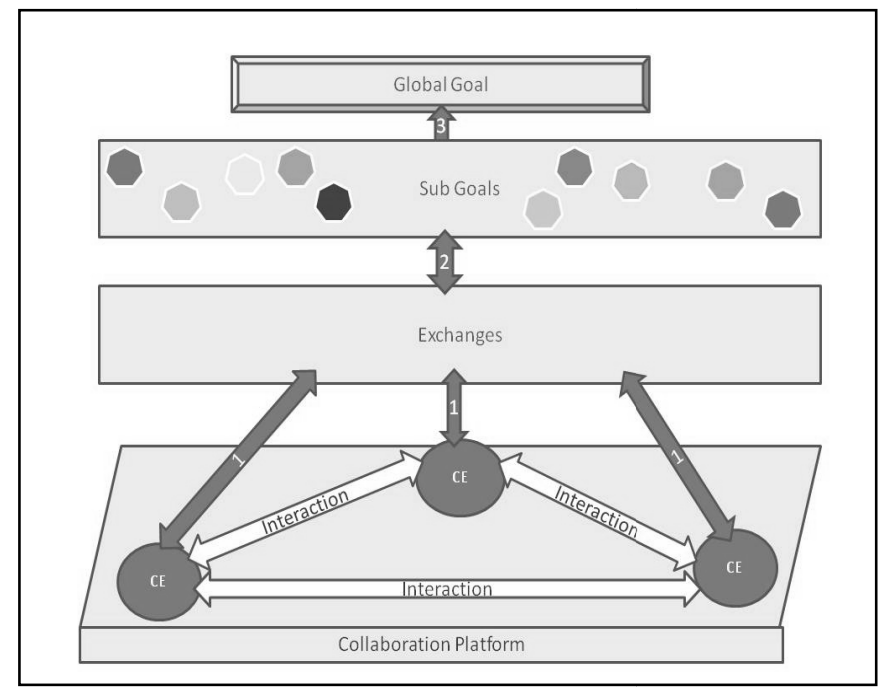

Fig. 1. Model of e-collaboration scenarios

In fact, the collaboration platform supports interactions between collaborators. These interactions produce interesting exchanges allowing the satisfaction of collaborator's sub goals and so the global goal achievement. This last aspect can be considered as the e-collaboration kernel and deserves a more elaborated description.

\section{Interaction details}

The carried out observation of e-collaboration scenarios has shown the existence of a common exchange process. In fact, in order to communicate with Collaborator $Y$, Collaborator $\mathrm{X}$ has to interact with its computer supposed to communicate with the receiver's one. To access to the sent information, collaborator $\mathrm{Y}$ needs to communicate on his turn with his computer. From this explication, three interaction types appear as shown in Fig. 2: Computer to Computer Interaction, Collaborator to Computer Interaction, Collaborator to Collaborator Interaction [8][9].

Thanks to the scenario observation step, we were able to produce some abstractions. This work will be completed by a classification of the various e-collaboration cases. The obtained results will be a start point for constructing the reusable evaluation method.
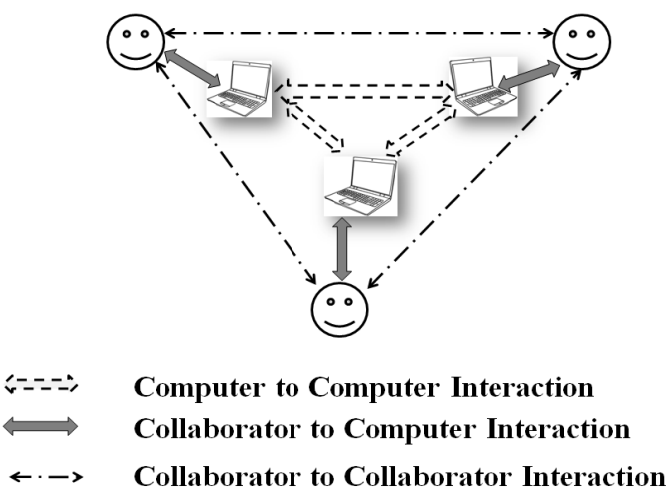

Fig. 2. Interaction view

\section{SCENARIO CLASSIFICATION}

As explained previously, e-collaboration scenarios are unlimited and their evaluation is strongly related to each particular context. But to give reusable evaluation solutions, we have to find a classification for these scenarios. As ecollaboration sessions have, in general, many goals and suppose the execution of many tasks; they contain many work steps. We distinguish two step types: collaborative and individual. Each step is related to the accomplishment of a sub goal and imposes the carrying out of one or many subtasks. Collaborative steps introduce usually communications between collaborators while individual ones consist in a single work. In the following, we propose a classification of collaborative steps. In this context, the word e-collaboration must be understood as an e-collaboration step.

In fact, e-collaboration classification can be performed regarding to resource exploitation:

- Collaboration on a common resource;

- Collaboration based on resource exchange.

The word "resource" denotes not only software and hardware resources but also exchanged knowledge, information and data that are less concrete but have the same importance.

For each class, two principal organizations are available:

- Peer to Peer organization: in which all the collaborators participate to tasks in an equal manner. None of the collaborators has dominance or influence over the others.

- Hierarchical organization: in which collaborators do not have the same responsibilities. One or many of them are supposed to treat received information from other members. The treatment can have different forms like correction, selection, consolidation and many objectives like making decisions and supervising obtained results. 
If we combine the two distinct classes with both organizations, we obtain four categories of e-collaborations like shown in Fig. 3.

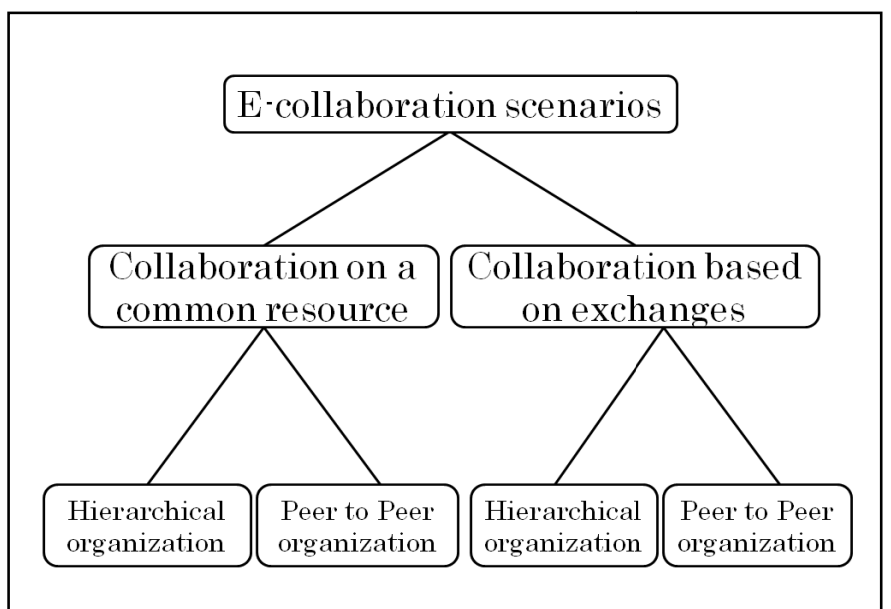

Fig. 3. Scenario classification

To make this classification clear, we give some examples:

- A classical vote scenario for making decision can be considered as a collaboration based on resource exchange with an hierarchical organization;

- The collaborative construction of a dictionary regrouping technical words of a particular domain belongs to the first class and is generally equally organized.

\section{EVALUATION METHOD}

In the previously presented interaction view, we believe that Collaborator to Collaborator Interaction is the most influential interaction type of e-collaboration and at the same time, we note that it is the less treated by evaluation works. For this reason, the evaluation method proposed in the following, will be focused on Collaborator to Collaborator Interaction. Contrarily to some other approaches that are based on particular scenarios [15], this one is judged useful because it permits to produce a reusable evaluation method. The proposed method is composed by two evaluation levels. The first focuses on detecting the unexpected e-collaboration results by observing the state of e-collaboration components cited in section IV and summarized in figure 1. Once the first level accomplished, the second one aims to explain the previously detected inconsistencies based on a validated error analysis method entitled CREAM (Cognitive Reliability Error Analysis Method) [11][13]. In the following, we detail the work to do for each evaluation level.

\section{A. First level}

In the beginning, let us recall e-collaboration elements that are: e-collaboration platform, e-collaborators, sub-goals and global goal. The values taken by the components of an ecollaboration scenario constitute its context.

Generally, in e-collaboration sessions, the expected context is not identical to the current one. Differences between the two contexts have necessarily effects on the obtained results. We propose, in this level, to identify the two contexts and to compare them. To do that, evaluator is supposed to recover information on real and expected contexts from an e-collaborator. To ensure having adequate details on the context's scenario, the contacted e-collaborator must have a global view on the progress of the scenario. We estimate that the collaborator's view on the scenario depends on its organization. If it is hierarchical, evaluator must communicate with an e-collaborator from the upper level of the hierarchy. And if it is organized in a peer to peer manner, any e-collaborator is supposed to keep the required information.

\section{B. Second level}

This level is concerned with the explanation of the previously detected inconsistencies. For this aim, we propose to apply the generic error analysis method called CREAM. In the literature, it has been shown that: compared to many other existing error analysis methods, CREAM has the advantage of implementing a highly efficient error analysis strategy [11]. It permits to deduce, after several iterations, the possible causes of unexpected results as well as the different links between the inconsistency and its antecedent. The CREAM method is composed of three principal steps.

The first step consists in a description of e-collaboration progress conditions through the notion of "Common Performance Conditions" designated also by CPC. The CPC are proposed to capture the situation key aspects and particularly the work conditions that may have an impact on the job progress. Each aspect of the CPC is qualified with some "descriptors" and their impact on performance. To guarantee the usability of this method, the number of proposed CPC has been limited.

The second step of the method consists in determining phenotypes (wrong actions) which describe behavior observations and classify them into the following types:

- Action at the wrong time (time, duration);

- Action of wrong type (distance, speed, direction, force);

- Action on the wrong object (neighbor, similar or unrelated);

- Action in the wrong place (sequence problems).

In the case of proposed e-collaboration evaluation method, phenotypes detection is the same as inconsistencies detection which has already been carried out in the first step. So, the application of CREAM is adapted to our context and we have only, in this evaluation second level, to classify the observed inconsistencies in the cited action types.

The third step focuses on identifying, by using "Common Performance Conditions", the causes of detected phenotypes. These causes are known under the name of genotypes. This final step is generally performed in several iterations. At each one, an effect and a cause are detected and the last cause becomes effect in the next iteration. This work provides causal links permitting to explain the occurrence of wrong action. In the original version of CREAM method, genotypes are classified in three categories as follows: Genotype related to the person $(\mathrm{P})$, Genotype related to technology $(\mathrm{T})$, genotype related to organization $(\mathrm{O})$. 
All the presented facts, antecedents and links between them can be schematized by a causal graph where nodes represent antecedents or facts and arcs describe the causal links. Such graph has the advantage of summing the different explications of a wrong action; but it doesn't show the most plausible one. For this, we were inspired by the work referenced by [14] that suggests assigning probabilities to each node of the graph. These probabilities or masses are computed using "the evidence theory" proposed by Dempster [12]. The end points of the graph represent explications of the phenotype in question.

\section{TEST SCENARIO}

To make more concrete the previous explained evaluation method, we propose to describe its application on the vote scenario described in section IV. This scenario starts when a notation problem is detected. The manager diffuses it to the BPMN group composed by members working on the improvement of this language; he determines a deadline of one week to receive responses. BPMN members are supposed to respond at the fixed delay. After a week, the manager collects the proposed solutions and broadcast them to another team of voters. The selection of solutions by vote is carried out in two cycles as shown in Fig. 4 and Fig.5.

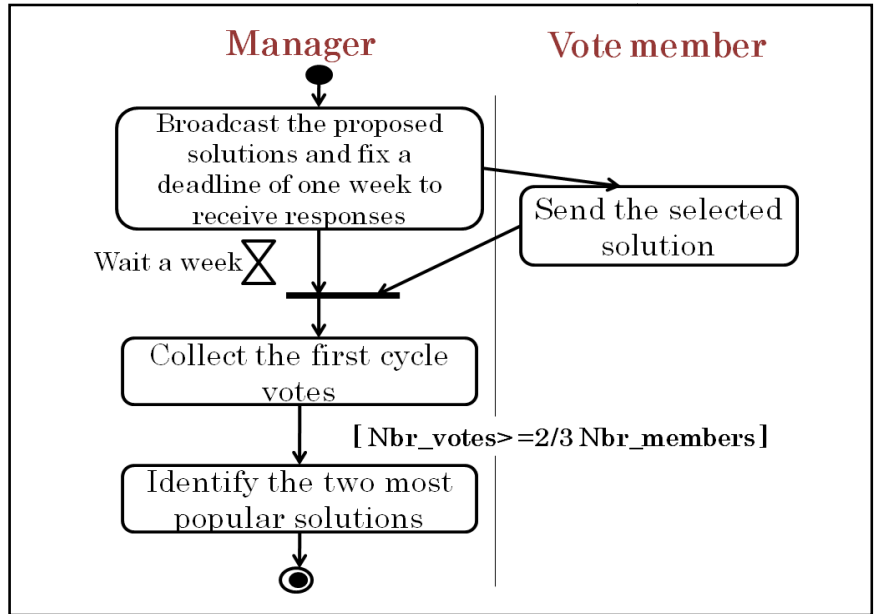

Fig. 4. Activity diagram of the first vote cycle

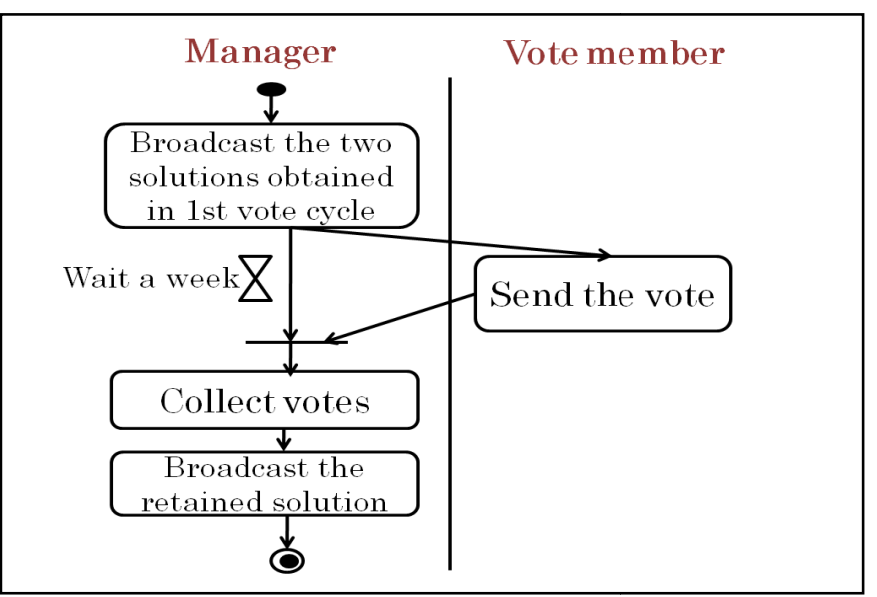

Fig. 5. Activity diagram of the second vote cycle
In the first cycle, the two most popular solutions are selected and in the second, one of them is retained.

\section{A. First evaluation level}

This scenario is in accordance with the previously proposed model; it corresponds to a "collaboration based on exchanges" and is hierarchically organized. In this level, evaluation is reduced to a comparison between the envisaged and real contexts. The compared values of e-collaboration components are grouped in table1 which shows that envisaged e-collaborators are present and communicate through the expected support. But two important sub-goals concerning the solution selection were not realized and stopped the scenario progress.

In fact, after an attentive observation of the activity diagram summarizing the first vote cycle; we can easily remark the existence of an important condition that is: [Nbr_votes $>=2 / 3 \mathrm{Nbr}$ members]. This condition imposes that the number of received votes is higher than two-thirds of the total number of vote members. The non accomplishment of this condition stops the vote scenario and this is what happened in this case. In the next evaluation level, we will try to find the eventual causes of the insufficient obtained number of votes.

Table 1- COMPARISON BETWEEN ENVISAGED AND EFFECTIVE ECOLLABORATION CONTEXTS

\begin{tabular}{|l|l|l|}
\hline & Envisaged components & Effective components \\
\hline $\begin{array}{l}\text { E- } \\
\text { collaborators }\end{array}$ & $\begin{array}{l}\text { Manager, 10 BPMN } \\
\text { members, 20 vote } \\
\text { members }\end{array}$ & $\begin{array}{l}\text { Manager, 10 BPMN } \\
\text { members, 20 vote } \\
\text { members }\end{array}$ \\
\hline $\begin{array}{l}\text { E- } \\
\text { collaboration } \\
\text { support }\end{array}$ & E-mail & E-mail \\
\hline Sub-goals & $\begin{array}{l}\text {-Recover solutions to the } \\
\text { detected notation } \\
\text { problem; } \\
\text {-Select the two popular } \\
\text { solutions; } \\
\text {-Retain one of the two } \\
\text { selected solutions }\end{array}$ & $\begin{array}{l}\text { A set of solutions is } \\
\text { recovered. }\end{array}$ \\
\hline Global goal & $\begin{array}{l}\text { Find solution to the } \\
\text { raised problem }\end{array}$ & $\begin{array}{l}\text { The goal is not } \\
\text { achieved }\end{array}$ \\
\hline
\end{tabular}

\section{B. Second level}

In this level, we started by determining the CPC by diffusing a questionnaire to all the e-collaborators. After that, the previously detected phenotype had to be classified. In fact, the insufficient number of votes expresses that several members haven't sent their choices in the specified delay. So this phenotype can be classified as an "action in a wrong time". At the final step and taking account of the CPC, explanations are generated permitting to construct the causal graph represented in Fig. 6. The obtained causal graph 6. Node masses are calculated in accordance to the formula of Dempster as follows.

To compute an end point mass, we have to start by calculate its consequence masses. This action is repeated until attaining the original antecedent that is the phenotype in question. 
Dempster's theory suggests that a consequence provides a part of its mass to each one of its antecedents according to the following formula:

$$
m(a)=p(C(a)) \times \sum_{\mathrm{b} \in \operatorname{Cons}(\mathrm{a})} \frac{\mathrm{m}(\mathrm{b})}{\sum_{\forall \mathrm{i} \in \mathrm{P}, \mathrm{T}, \mathrm{O}}\left(\mathrm{p}(\mathrm{i}) \times \mathrm{n}_{\mathrm{ib}}\right)}
$$

$\mathrm{m}(\mathrm{a})$ is the antecedent mass, $\mathrm{m}$ (phenotype $)=1$;

$\mathrm{C}(\mathrm{a})$ is the category of a;

Cons(a) is the set of consequences;

$p(i)$ is the weight of category i.

In this context, we consider that all antecedent categories are equiprobable. So: $\forall i, p(i)=0.33$

$\mathrm{n}_{\mathrm{ib}}$ is the number of antecedents of $\mathrm{b}$ belonging to category $\mathrm{i}$

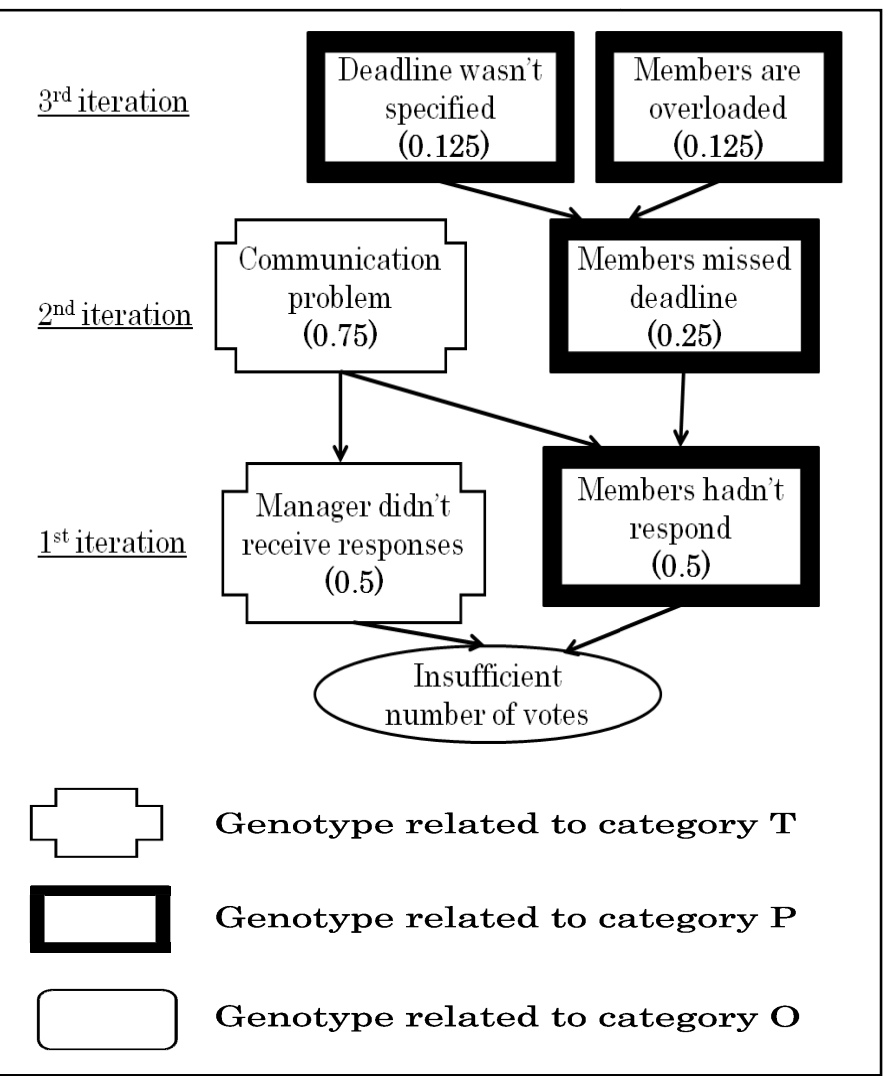

Fig. 6. The obtained causal graph

According to the presented graph and particularly to the calculated masses, we can deduce that the most plausible cause of the obtained insufficient number of votes is a communication problem. So, according to the proposed method, the problem is due to the used communication platform and it is recommended to verify its adequacy to avoid such problems in the future.

\section{CONCLUSION}

In this paper, we described the approach followed to provide a reusable evaluation method focused on a human reliability analysis rather than a classical metric computation. To attain this goal, we adopted a bottom-up process starting from the observation of some real scenarios in order to perform abstractions enabling a more formalized vision of ecollaboration sessions. We extracted an e-collaboration model summarizing common components between all the scenarios; the importance of interactions prompted us to dedicate a description part showing the existence of three different interaction types supporting collaborator's exchanges. As our work is based on scenario analysis and as e-collaboration cases are many; a classification was proposed. This work conducted us to construct the evaluation method based on two levels: problem detection at a first step and an explanation of its causes at a second step. The proposed method is currently in a validation phase that has the purpose to associate classes of e-collaboration scenarios with protocols dedicated to the evaluation of their effectiveness.

\section{REFERENCES}

[1] R. Basili and H. D. Rombach, "Tailoring the software process to project goals and environments", Proceedings of the 9th International Conference on Software Engineering, pp. 345-357, 1987.

[2] L. Damianos, L. Hirschman, R. Kozierok, J. Kurtz, A. Greenberg, K. Walls, S. Laskowski and J. Scholtz, "Evaluation for collaborative systems", ACM Computing Surveys, Vol. 31, No. 2, pp 15-26, 1999.

[3] W. M. Post, M. A. A. Huis in 't Veld and S. A. A. van den Boogaard, "Evaluating meeting support tools", Personal and Ubiquitous Computing - Special Issue: User-centred design and evaluation of ubiquitous groupware; Springer-Verlag London, UK; Volume 12 - Issue 3, January 2008.

[4] M. Eisenstadt, J. Komzak, and S. A. Cerri, "Peer conversations for elearning in the grid", 1st International ELeGI Conference on Advanced Technology for Enhanced Learning, Vico Equense (Naples), Italy, 2005.

[5] Business Process Model and Notation, V1.1, January 2008 (Standard document URL: http://www.omg.org/spec/BPMN/1.1/PDF)

[6] M. P. Steves and J. Scholtz, "A framework for evaluating collaborative systems in the real world." Proceedings of the 38th Annual Hawaii International Conference, 2005.

[7] Westphal, K. D. Thoben, and M. Seifert, "Measuring collaboration performance in virtual organizations", Establishing The Foundation Of Collaborative Networks, Portugal, Springer Verlag, pp.33-42, 2007.

[8] R. Chebil, W. Lejouad Chaari and S. A. Cerri, "An E-Collaboration New Vision and Its Effects on Performance Evaluation", International Journal of Computer Information Systems and Industrial Management Application, Volume 3, pp. 560 -567, 2011.

[9] R. Chebil, W. Lejouad Chaari and S. A. Cerri, "Performance Evaluation of E-Collaboration", Proceedings of the IADIS International Conference on Collaborative Technologies, pp. 163-167, 2010.

[10] N. Kock and J. Nosek. "Expanding the boundaries of e-collaboration". Professional Communication, IEEE Transactions, pp. 1-9, 2008.

[11] Erik Hollnagel, "Cognitive Reliability and Error Analysis Method", Elsevier Science, 1998.

[12] P. Dempster, "Upper and lower probabilities induced by a multivalued mapping", The Annals of Mathematical Statistics, Vol. 38, pp. 325-339, 1967.

[13] He. XuHong, "A simplified cream prospective quantification process and its application”, Reliability Engineering \& Safety System, Vol. 93, pp.298-306, 2005.

[14] Naïma El-Kechaï, « Christophe Després, Rechercher les causes erronées $\mathrm{du}$ formé pour les proposer au formateur», Environnements informatiques pour l'apprentissage humain, Lausanne 2007.

[15] Y. B. Salman, I. F. Ince, J. Y. Kim, H. I. Cheng, and M. E. Yildirim, "Participatory design and evaluation of e-learning system for korean language training", Proceedings of the $2^{\text {nd }}$ International Conference on Interaction Sciences : Information Technology, Culture and Human (ICIS'09), pp. 312-319, 2009. 